\title{
观察利拉鲁肽对老年2型糖尿病患者糖脂代谢、胰岛功 能及骨密度的影响
}

\author{
马 季* \\ 甘肃省嘉峪关市中医医院，甘肃 735100
}

摘 要：目的：对老年T2DM患者使用利拉鲁肽治疗，并分析其应用效果。方法：选取2018年1月-2020年1月， 在我院治疗的 86 例老年T2DM患者, 将其分为两组。对照组43例, 使用二甲双胍治疗; 观察组43例, 使用利拉鲁肽治 疗。对比两组患者的糖脂代谢指标、骨密度、胰岛功能等指标。结果：两组患者的FBG、HbA1c水平无明显差异 $(P>$ $0.05)$; 观察组患者的TG、TC水平明显低于对照组 $(P<0.05)$; 观察组患者的FC-P、FINS、HOMA- $\beta$ 水平明显高于 对照组, HOMA-IR水平明显低于对照组 $(P<0.05)$; 观察组患者的BGP、BMD水平明显高于对照组 $(P<0.05)$ 。 结论：对老年T2DM患者使用利拉鲁肽治疗可有效改善患者血糖水平、脂代谢、胰岛功能及骨密度状态，具有较高的 推广价值。

关键词：胰岛功能；骨密度；糖脂代谢；利拉鲁肽

\section{一、前言}

老年人是 2 型糖尿病 ( T2DM ) 的主要发病人群, 且研究发现, 老年T2DM患者往往血糖控制不佳, 且随着病程的 延长, 容易出现严重的心血管疾病并发症, 严重影响患者的身心健康及生活质量 ${ }^{[1]}$ 。同时, 老年T2DM患者多存在胰 岛 $\beta$ 细胞功能受损、血管内皮细胞功能紊乱情况, 而后者是诱发心脑血管疾病、动脉弹样硬化的重要危险因素。由此 可见, 在老年T2DM患者治疗期间, 改善患者胰岛 $\beta$ 细胞功能非常重要。利拉鲁肽属于新一代降糖药物, 是一种胰高血 糖素样肽-1（GLP-1）类似物, 具有良好的控制血糖、调节血脂、改善胰岛 $\beta$ 细胞功能、降低体重等效果, 但在利拉鲁 肽对患者骨密度影响方面, 目前相关研究较少 ${ }^{[2]}$ 。考虑到老年患者的特殊性, 在选择治疗药物时, 必须充分考虑其对 骨密度的影响。针对这种情况，本文将对老年T2DM患者使用利拉鲁肽治疗，并分析其应用效果，现报道如下。

\section{二、资料与方法}

(一) 一般资料

选取2018年1月一2020年1月，在我院治疗的86例老年T2DM患者。

1. 纳人标准

（1）根据《中国2型糖尿病防治指南》 ${ }^{[3]}$, 明确诊断为T2DM。

(2) 初发患者, 人组前未使用降糖药物治疗。

( 3 ) 年龄 $>60$ 岁。

(4) 病历资料完整, 遵医行为良好, 已签署知情同意, 经过医院伦理委员会批准。

2. 排除标准

( 1 ） 1 型糖尿病患者。

(2) 其他原因所致高血糖患者。

（３）合并相关急性、慢性并发症患者。

(4) 存在内分泌疾病、肝肾功能异常、心脑血管疾病患者等。

采取随机数字表法, 将其分为两组。观察组43例, 男性21例, 女性22例, 年龄63 74岁, 平均 $(66.75 \pm 4.36)$ 岁, 空腹血糖 $\left(\mathrm{FPG}\right.$ ) 为 $7.24 \sim 10.25 \mathrm{mmol} / \mathrm{L}$, 平均 $(8.84 \pm 0.98) \mathrm{mmol} / \mathrm{L}$, 体重指数 (BMI) 为 $22.41 \sim 27.89 \mathrm{~kg} / \mathrm{m}^{2}$, 平均

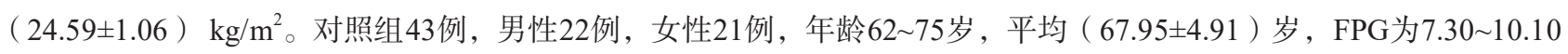

*通讯作者: 马季, 1973 年09, 男, 回族, 甘肃嘉峪关人, 就职于甘肃省嘉峪关市中医医院, 副主任医师, 本 科。研究方向: 内分泌方面。 
$\mathrm{mmol} / \mathrm{L}$, 平均 $(8.79 \pm 0.91) \mathrm{mmol} / \mathrm{L}$, BMI为 $22.50 \sim 28.01 \mathrm{~kg} / \mathrm{m}^{2}$, 平均 $(24.64 \pm 1.09) \mathrm{kg} / \mathrm{m}^{2}$ 。

(二) 方法

所有患者均接受饮食指导，制定个体化糖尿病饮食处方；给予患者运动指导， $20 \mathrm{~min} /$ 次，3 次/d。同时，对照组 患者使用二甲双胍 (贵州天安药业股份有限公司, 国药准字H20073382) 治疗, 口服, 0.25 0.5 g/次, 3 4 次/d。观察 组患者使用利拉鲁肽 ( S20160004, Novo Nordisk A/S ) 治疗, 皮下注射, 初始剂量 $0.6 \mathrm{mg} /$ 次, 1 次/d, 随后调整为 1.2 $\mathrm{mg} /$ 次， 1 次 $/ \mathrm{d}$ 。两组患者均治疗 4 周，治疗期间定期进行血糖值监测。

（三）评价标准

1. 对比两组患者的糖脂代谢指标, 包括糖化血红蛋白（ HbA1c）、空腹血糖（FBG）、甘油三酯（TG）、总胆 固醇 $(\mathrm{TC})$ 等。

2. 对比两组患者胰岛功能指标, 包括空腹C肽 (FC-P) 、空腹胰岛素（FINS）、胰岛素抵抗指数（HOMAIR）、胰岛素分泌指数 ( HOMA- $\beta$ ) 等。

3. 对比两组患者骨密度指标, 包括骨钻素 (BGP) 及骨密度 (BMD) 指标, BGP通过采取放免法检测, BMD使 用双能X线骨密度仪检测，测量部位为第1 4腰椎。

(四) 统计学方法

采取SPSS22.0软件处理, 计数资料使用 ( \% ) 表示, 计量资料使用 $\left(\chi^{2} \pm s\right)$ ，进行 $\chi^{2}$ 或 $t$ 检验。 $P<0.05$ 表示差 异, 有统计学意义。

\section{三、结果}

（一）糖脂代谢指标对比

两组患者的FBG、HbA1c水平无明显差异 $(P>0.05)$; 观察组患者的 $\mathrm{TG} 、 \mathrm{TC}$ 水平明显低于对照组 $(P<0.05)$, 见表1。

表1 两组患者糖脂代谢指标对比 $\left(n=43, \chi^{2} \pm s\right)$

\begin{tabular}{cccccc}
\hline 组别 & 阶段 & FBG $(\mathrm{mmol} / \mathrm{L})$ & $\mathrm{HbA1 \textrm {c }}(\%)$ & $\mathrm{TG}(\mathrm{mmol} / \mathrm{L})$ & $\mathrm{TC}(\mathrm{mmol} / \mathrm{L})$ \\
\hline \multirow{2}{*}{ 观察组 } & 治疗前 & $11.28 \pm 3.68$ & $10.22 \pm 1.33$ & $2.77 \pm 0.89$ & $5.88 \pm 1.33$ \\
& 治疗后 & $5.67 \pm 0.92$ & $7.08 \pm 1.10$ & $1.62 \pm 0.30 \mathrm{a}$ & $4.02 \pm 0.35 \mathrm{a}$ \\
\multirow{2}{*}{ 对照组 } & 治疗前 & $11.30 \pm 3.70$ & $10.27 \pm 1.29$ & $2.79 \pm 0.90$ & $5.89 \pm 1.26$ \\
& 治疗后 & $5.81 \pm 0.80$ & $7.20 \pm 1.11$ & $1.81 \pm 0.28$ & $4.80 \pm 0.41$ \\
\hline
\end{tabular}

与对照组对比: ${ }^{a} P<0.05$ 。

（二）胰岛功能对比

治疗后，观察组患者的FC-P、FINS、HOMA- $\beta$ 水平明显高于对照组，HOMA-IR水平明显低于对照组 $(P<$ $0.05 ） ， 见$ 表2。

表2 两组患者胰岛功能对比 $\left(n=43, \chi^{2} \pm s\right)$

\begin{tabular}{cccccc}
\hline 组别 & 阶段 & FC-P $(\mu \mathrm{g} / \mathrm{L})$ & FINS ( IU/L ) & HOMA-IR & HOMA- $\beta$ \\
\hline \multirow{2}{*}{ 观察组 } & 治疗前 & $0.84 \pm 0.30$ & $12.90 \pm 3.36$ & $5.70 \pm 0.72$ & $28.72 \pm 8.49$ \\
& 治疗后 & $1.89 \pm 0.33 \mathrm{~b}$ & $23.83 \pm 1.25 \mathrm{~b}$ & $3.28 \pm 0.37 \mathrm{~b}$ & $87.48 \pm 8.58 \mathrm{~b}$ \\
\multirow{2}{*}{ 对照组 } & 治疗前 & $0.85 \pm 0.29$ & $12.89 \pm 3.42$ & $5.66 \pm 0.80$ & $27.79 \pm 7.06$ \\
& 治疗后 & $1.56 \pm 0.28$ & $18.78 \pm 1.09$ & $4.23 \pm 0.40$ & $77.78 \pm 9.02$
\end{tabular}

与对照组对比: ${ }^{\mathrm{b}} P<0.05$ 。

(三) 骨密度对比

表3 两组患者骨密度对比 $\left(n=43, \chi^{2} \pm s\right)$

\begin{tabular}{cccc}
\hline 组别 & 阶段 & BGP $(\mu \mathrm{g} / \mathrm{L})$ & BMD $\left(\mathrm{g} / \mathrm{cm}^{2}\right)$ \\
\hline \multirow{2}{*}{ 观察组 } & 治疗前 & $5.79 \pm 0.89$ & $1.65 \pm 0.17$ \\
& 治疗后 & $16.77 \pm 1.79 \mathrm{c}$ & $2.77 \pm 0.58 \mathrm{c}$ \\
对照组 & 治疗前 & $5.80 \pm 0.90$ & $1.67 \pm 0.20$ \\
& 治疗后 & $6.20 \pm 1.20$ & $1.65 \pm 0.60$ \\
\hline
\end{tabular}


与对照组对比: ${ }^{\mathrm{c}} P<0.05$ 。

治疗后，观察组患者的BGP、BMD水平明显高于对照组 $(P<0.05)$ ，见表3。

四、讨论

我国糖尿病患病人口基础较大, 且 $90 \%$ 以上患者均为 $\mathrm{T} 2 \mathrm{DM}$ 患者, 老年患者占比较高。研究发现, 与中青年患 者相比, 老年T2DM患者多伴有血管内皮功能损伤, 极易诱发心脑血管疾病、动脉籿样硬化 ${ }^{[4]}$ 。同时, 随着病程的延 长、病情的进展, 胰岛 $\beta$ 细胞功能也会逐步下降, 影响血糖控制的稳定性。此外, 随着内源性胰岛素分泌量的不断下 降, 大部分患者在发病 10 年内会出现单纯口服降糖药物效果下降的情况, 需要联合胰岛素治疗, 但该治疗方案在改善 患者胰岛 $\beta$ 细胞功能、血管内皮功能方面效果不理想。近年来，利拉鲁肽在临床上逐步得到应用，收到了显著成果， 为老年T2DM患者的临床治疗提供了新的途径 ${ }^{[5]}$ 。

GLP-1是回肠、结肠、空肠等L细胞合成的物质, 在进餐后, GLP-1分泌增加, 并会刺激胰岛 $\beta$ 细胞合成, 增加胰 岛素的分泌。同时，GLP-1还能够对胃酸分泌、胰高血糖素产生抑制作用，延缓胃排空，提高胰岛素敏感性。此外， GLP-1还能够抑制摄食中枢，减少摄食，从而调节血糖水平 ${ }^{[6]}$ 。作为GLP-1受体激动剂，使用利拉鲁肽治疗后，可上调 GLP-1受体, 从而使GLP-1发挥作用, 达到控制血糖、减轻体重、增加胰岛素分泌等效果。在本次研究中, 两组患者 的FBG、 $\mathrm{HbA1c}$ 水平无明显差异 $(P>0.05)$; 观察组患者的TG、TC水平明显低于对照组 $(P<0.05)$; 观察组患者的 FC-P、FINS、HOMA- $\beta$ 水平明显高于对照组, HOMA-IR水平明显低于对照组（ $P<0.05 ）$, 表明两种药物的降糖效果 相当，但利拉鲁肽对血脂、胰岛功能改善效果更明显。推测与利拉鲁肽增强胰岛素敏感性、抑制胰高血糖素分泌、刺 激胰岛素分泌、减轻体重等作用机制有关。以往有报道显示 ${ }^{[7]}$ ，对老年T2DM患者使用利拉鲁肽治疗后患者FPG、餐后 $2 \mathrm{~h}$ 血糖（2h PBG）、HbA1c等血糖指标以及FINS、HOMA-IR、HOMA- $\beta$ 等胰岛功能指标改善效果更好, 与本次研究 结果基本相符。此外, 在本次研究中, 观察组患者的BGP、BMD水平明显高于对照组 $(P<0.05)$, 表明利拉鲁肽可 更好地改善患者骨密度水平，而二甲双胍对患者骨密度无明显改善效果。

分析其改善骨密度的机制, 动物实验显示, 利拉鲁肽可使去卵巢大鼠骨合成增加, 激活Wtn通路, 可减少骨丢 失。也有研究显示 ${ }^{[8]}$, 对于新诊断T2DM患者, 利拉鲁肽、二甲双胍对骨密度影响均不显著, 但利拉鲁肽可降低血清 $\beta-C T x$ 水平。因此, 对于老年患者、体重下降的肥胖妇女, 可使用利拉鲁肽治疗, 促进骨形成, 通过调节肠道钙磷代 谢可减少骨流失。应注意的是, 由于利拉鲁肽会影响胃肠蠕动及胃排空，因此可能引起恶心、呕吐等表现，部分患者 可能出现低血糖，应注意调整剂量，提高用药安全性。

综上所述，对老年T2DM患者使用利拉鲁肽治疗可有效改善患者血糖水平、脂代谢、胰岛功能及骨密度状态，具 有较高的推广价值。

\section{参考文献:}

[1]林园,冯利.利拉鲁肽联合二甲双胍对老年2型糖尿病患者血清Hcy、CysC、hs-CRP水平及糖脂代谢的影响 [J].中 国老年学杂志, 2020,40(03):502-504.

[2]田利华,薛亚男,涂强.幽门螺杆菌感染与非酒精性脂肪肝对2型糖尿病患者糖脂代谢的影响 [J]. 检验医学与临床, 2020,17(02):237-240.

[3]李爽, 沈莺, 赵娜. 西药联合安糖饮对脾虚痰湿型2型糖尿病42例胰岛素抵抗及糖脂代谢的影响 [J]. 中国民族民间医 药, 2020,29(01):97-99.

[4]陆梓华, 吕雄, 曹明满, 毕建璐, 黄䎦丽. 舒正颗粒对2型糖尿病大鼠糖脂代谢异常PI3K/AKT信号通路的影响[J].广州 中医药大学学报, 2020,37(01):128-134.

[5]马军民, 李伟, 何泽来.5 种肿瘤标志物与 2 型糖尿病患者糖脂代谢的相关性分析 [J]. 晥南医学院学报, 2019,38(06):528-531.

[6]梁敏, 王海牛, 黄鹏, 朱玮华, 李顺昌.抗阻运动对2型糖尿病糖脂代谢异常患者干预效果的系统综述和Meta分析 [J]. 中 国组织工程研究, 2019,23(35):5718-5726.

[7]李芳.观察利拉鲁肽联合二甲双胍治疗老年2型糖尿病的临床效果[J].中国现代药物应用, 2019,13(08):82-83.

[8] 曾海勇, 李海燕, 周丽明, 李明政, 刘雪婷.地特胰岛素联合二甲双胍、利拉鲁肽治疗老年2型糖尿病的疗效及安全性 分析[J].现代诊断与治疗, 2018,29(15):2369-2371. 